Cultures \& Conflits

28 | hiver 1997

Interpréter l'Europe

\title{
La crise du service public en France : l'Europe comme catalyseur
}

Luc Rouban

\section{OpenEdition}

1 Journals

\section{Édition électronique}

URL : http://journals.openedition.org/conflits/383

DOI : $10.4000 /$ conflits.383

ISSN : $1777-5345$

Éditeur :

CCLS - Centre d'études sur les conflits lilberté et sécurité, L'Harmattan

Édition imprimée

Date de publication : 15 janvier 1997

ISSN : 1157-996X

Référence électronique

Luc Rouban, "La crise du service public en France : l'Europe comme catalyseur », Cultures \& Conflits

[En ligne], 28 | hiver 1997, mis en ligne le 16 mars 2006, consulté le 30 mars 2021. URL : http://

journals.openedition.org/conflits/383 ; DOI : https://doi.org/10.4000/conflits.383

Ce document a été généré automatiquement le 30 mars 2021.

Creative Commons License 


\title{
La crise du service public en France : l'Europe comme catalyseur
}

\author{
Luc Rouban
}

2 Conceptualiser l'Europe revient à la saisir dans ses manifestations. L'objet " Europe " se dérobe au regard lorsqu'on le fixe, pour ne laisser qu'une impression rétinienne faite de comitologie et de programmes techniques. La logique européenne échappe donc à l'observateur ou s'impose sous le seul aspect d'une absence du politique ou du social. Le politiste doit donc user de l'Europe comme l'orpailleur du mercure : il s'agit d'un révélateur de structures cachées. L'Europe n'est donc plus un objet étranger, marqué d'une altérité à laquelle on opposerait la spécificité française si bien connue qu'elle même ne se donne à voir aujourd'hui qu'au détour d'une réflexion sur l'Europe... Force est de constater que l'action publique est imprégnée d'Europe et que la construction européenne reste marquée par la culture des divers Etats qui lui ont donné naissance. La difficulté, bien entendu, est que l'on va souvent trop vite en besogne alors même que bien des aspects de la situation politique française ont pu évoluer ou rester dans l'ombre. A tout prendre, lorsqu'on examine la question du service public, il apparaît que sa dimension nationale est sans doute bien moins connue que l'ensemble des mesures prises par la Commission sur les services économiques d'intérêt général ${ }^{2}$. En examinant les services publics à la lumière de l'Europe et l'Europe au regard des services publics, il faut donc faire preuve de prudence car les amalgames ne manquent pas: confusion entre la déréglementation mondiale et l'action des instances européennes, mélange des genres (en quoi le transport est-il assimilable à l'échange d'information ou d'électricité ?), et, bien entendu, tours de passe-passe idéologiques permettant de substituer la notion de service public à celle d'Etat ou de république ${ }^{3}$. On partira de l'hypothèse que l'interaction de l'Europe et des services publics nationaux participe d'une recomposition politique générale. Les services publics offrent un terrain d'essai ou d'exploration pour de nouvelles configurations conceptuelles mais aussi pour de nouveaux modèles institutionnels. La construction européenne impliquet-elle seulement un rite de passage, un apprentissage de nouvelles normes, ou bien 
conduit-elle à rompre avec des pratiques sociales et des univers de sens chargés d'histoire? Peut-on ajouter une dimension supplémentaire à l'action publique sans bouleverser la culture politique nationale? Les modes d'organisation sociale en France, qui appellent la distinction entre secteur public et secteur privé, peuvent-ils résister aux nouveaux jeux stratégiques d'une déréglementation contrôlée? La question du service public face à l'Europe ne se pose pas en France comme elle a pu se poser en Grande-Bretagne, où ils ont été soumis à une vague sans précédent de privatisation depuis 1979, ou en Italie, où leur critique s'est appuyée sur une crise plus générale de la classe politique depuis le gouvernement Amato. Le service public reste foncièrement attaché à la définition de l'identité nationale française et toute remise en cause est alors perçue comme une question politique touchant au moins autant le rôle social du secteur public que son efficacité économique. Une chaîne logique s'établit donc très vite entre la déréglementation, l'Europe et la réforme de l'Etat ou du secteur public, multipliant les enjeux et traversant les clivages partisans. Depuis 1996, les principaux partis politiques français semblent se rétracter face à la construction européenne ou bien posent des conditions quant à sa poursuite. Celle-ci leur semble désormais plus riche en conflits potentiels qu'en gains électoraux, ce que vient confirmer la campagne des législatives de 1997. De fait, l'avenir de l'Union ne peut plus désormais se réduire à un consensus mou entretenu par un discours pédagogue plus ou moins iréniste. L'Europe implique des changements où se dévoilent des rapports de force que les partis politiques ne maitrisent pas. C'est en cela que la crise de 1995 peut être riche d'enseignements et révéler la nature des enjeux. La thèse que l'on soutiendra ici est qu'il faut se garder d'entériner l'un des deux grands récits mythiques permettant d'expliquer la construction européenne : celle-ci est en effet présentée soit comme " l'Europe de Maastricht ", c'est-à-dire comme une menace externe, indépendante de la volonté des Etats-Nations, statue du Commandeur qui viendrait imposer un ordre supérieur; soit comme le dévoilement d'une " gouvernance ", c'est-à-dire d'une transformation de l'ordre politique remplaçant le pouvoir unilatéral et centralisé par une multiplication de centres de décision plus ou moins autonomes trouvant des terrains d'arrangements provisoires ${ }^{4}$. Ces deux récits peuvent être critiqués car ils semblent très globalisants, faisant état d'un ordre déjà-là, d'une intelligibilité pleine et entière d'un processus complexe dont on connaîtrait à l'avance le point d'achèvement. La construction de l'Union pose un vrai problème théorique et méthodologique car les conclusions sont souvent tirées vers l'un ou l'autre modèle d'interprétation, en fonction des terrains de recherche. Le degré de conflictualité, la capacité des acteurs d'agréger des demandes divergentes ou éclatées, la superposition des centres de pouvoir et l'effet de surimpression optique qui s'en dégage, varient considérablement. Les conflits engendrés par la transformation des services publics doivent eux aussi être passés au crible de l'analyse critique, car les modèles d'interprétation que l'on a en stock peuvent très bien recouvrir une mutation politique d'un autre ordre. Le conflit de 1995 et l'utilité de l'EDM La question des services publics est placée au cœur des interrogations portant sur la construction européenne. En premier lieu, parce qu'elle vient illustrer le débat entre la déréglementation, qui serait l'œuvre de l'Union européenne, et la persistance d'une spécificité française séculairement attachée à un grand secteur public fait de monopoles et de prérogatives exorbitantes du droit commun, pour emprunter la langue des juristes. Les services publics se trouvent ainsi placés au centre de conflits sociaux et politiques qui ont pu trouver leur expression, bien que fort confuse, dans la crise de novembre-décembre 1995. Mais, au-delà de tous 
les discours que l'on a pu tenir sur cette crise, il semble bien que cette confrontation ne se réduise pas au choix du marché contre l'Etat ou de l'entreprise privée contre l'entreprise publique. Au fond, tout se passe comme si l'ouverture à la libre concurrence, qui constitue tout de même la raison d'être historique de l'Europe, était devenue le vecteur d'une autre évolution, sans doute imprécise et bien floue, mais touchant à la racine même du modèle politique français. La question pourrait être alors présentée ainsi: l'Etat français, après bien des années de controverses, a fini par accéder au statut de véritable Etat de droit mais au prix de compromis fragiles entre un régime politique profondément et héréditairement notabiliaire et un système social qui s'est émancipé et pluralisé. L'Europe vient remettre en cause cet équilibre instable entre l'autorité politique, qui ne s'est pas réellement démocratisée, et une société fragilisée par une crise économique de longue durée venant créer de nouvelles inégalités. Dans la crise de 1995, se joue essentiellement une crise de l'autorité : de l'autorité gouvernementale, accusée de ne pas négocier, de l'autorité " managériale " que la direction de la SNCF ou de la RATP pouvait avoir sur son personnel, critique diffuse du pouvoir " technocrate ", mais aussi de l'autorité syndicale, le front commun débouchant vite sur l'opposition de FO et de la CFDT, les centrales perdant vite le contrôle de la situation au profit de SUD et de la mouvance autogestionnaire. En symétrie, la dimension conviviale, voire festive des manifestations de 1995 vient souligner la recherche de solidarité en opposition à ce qui paraît être l'application aveugle d'une logique gestionnaire. Dans ce contexte, l'argument " européen " vient en contrepoint : l'Europe est celle de ces technocrates qui ouvrent les services publics aux vents de la mondialisation. La contradiction des argumentaires ne doit cependant pas cacher le registre sur lequel se déploie ce conflit : une question de justice sociale (faut-il ou non aligner les régimes de retraite des salariés du secteur public sur ceux du secteur privé et mettre fin aux régimes spéciaux de certains services publics?), qui aurait sans doute été réglée sans trop de débats dans d'autres systèmes politiques européens, devient le fer de lance d'une crise grave, menaçant le gouvernement et imposant à la CIG d'inscrire la question sociale à l'ordre du jour. Ce conflit est révélateur de la situation française vis-à-vis de l'Europe puisque des mesures autrement dures ont été prises partout ailleurs pour aligner les systèmes de fonction publique sur le droit commun du travail sans que des conflits de cette ampleur en aient résultés. Plus généralement, la France peut s'observer au miroir de l'Europe, qui devient ainsi le catalyseur de défauts structurels que les différents gouvernements qui se succèdent depuis 1981 n'ont pu corriger, piégés alternativement par les besoins de la légitimation (la gauche entérinant l'appareil institutionnel de la Ve République et inscrivant la décentralisation dans une logique purement notabiliaire) ou par les nécessités du compromis social (la droite maintenant une tradition interventionniste propice aux corporatismes). Le conflit de 1995 voit donc s'opérer une série de glissements discursifs mais aussi stratégiques qui conduisent progressivement du débat sur la retraite des cheminots au débat plus général sur " le plan Juppé " et la conduite de la modernisation économique de la société française. De ces glissements, un certain nombre d'interprétations ont été proposées qui sont loin d'être convaincantes :

- la crise de 1995 ne constitue certainement pas un mouvement social. Elle s'inscrit dans l'univers politique des services publics et ne se diffuse pas aux salariés du secteur privé. L'idée d'une grève par délégation est séduisante mais repose sur une interprétation un peu spécieuse, et largement relayée par les leaders de la grève, selon laquelle la neutralité sinon la sympathie des usagers, qui est un fait avéré, serait un 
blanc-seing donné aux grévistes. Un mouvement social aurait exigé des mobilisations intersectorielles qui ne se sont pas produites ${ }^{6} ;$. Et si les sondages d'opinion indiquent bien que la majorité des Français avaient de la sympathie pour les revendications des grévistes, c'est bien parce que cette grève venait contester le passage à la rigueur et le changement de cap de la politique présidentielle annoncé en octobre $1995^{7}$

- néanmoins, il est vrai que le conflit s'est organisé contre une culture économiste et managériale stigmatisée à travers la dénonciation du " plan Juppé ", et, très précisément, dans le refus des normes de productivité imposées par le projet de contrat de plan entre l'Etat et la SNCF qui prévoyait de réduire les déficits chroniques de l'entreprise publique. On voit se développer durant la crise de 1995 une analyse de l'Europe dans les termes d'un spill-over négatif, c'est-à-dire d'une contrainte agissant mécaniquement comme un engrenage puisqu'il permet de passer par transduction des critères de convergence pour la réalisation de la monnaie unique à la mise en œuvre d'une pensée unique gestionnaire puis, par voie de conséquence, à toute la gamme des contraintes professionnelles et sociales ; - la dénonciation de " l'Europe de Maastricht " (objet intellectuel que l'on nommera ici l'EDM) reste généralement au second plan mais justifie en négatif les revendications. L'EDM devient la synthèse de tous les refus : refus de l'économisme, des contraintes de gestion, de l'anomie sociale ou de la " trahison " de la gauche pro-européenne ${ }^{8}$. L'EDM n'est d'ailleurs qu'une des figures du libéralisme puisqu'elle inclut aussi bien la mondialisation que le travail administratif réalisé par les services de la Commission 9. L'EDM est alors critiquée pour sa dimension élitiste : la rupture entre le " peuple " et les " élites " est d'ailleurs présentée comme la grande explication du conflit de 1995, rupture que l'on décline sur la gamme de l'incapacité du gouvernement Juppé de communiquer ou sur celle de la pensée-Michel-Crozier des " élites incapables de gérer un pays parce que mal formées et vivant dans leur tour d'ivoire ", et de citer, bien entendu, l'ENA ou les grandes écoles qui seraient à l'origine de tous les maux. L'EDM est donc chargée d'une très forte connotation populiste, qui voit se rejoindre, dans une commune dénonciation des élites, les partisans du PCF et du Front National autour d'une même idéologie territoriale ${ }^{10}$. La redéfinition de la notion de territoire, déconnectée d'une analyse sérieuse des flux économiques ou géographiques liés à la mondialisation, offre la base conceptuelle sur laquelle peut se développer la critique de l'EDM autour des notions d'identité nationale ou de spécificité française (le " service public à la française "). Le thème du " vivre et travailler au pays " rejoint les revendications émanant des cheminots pour une part plus active dans la marche de leur entreprise comme la critique des délocalisations industrielles. L'EtatNation lui-même n'est plus conçu dans la perspective d'une construction démocratique active mais soit dans celle d'une lutte entre les " énarques " et le peuple, nouvelle expression de la lutte des classes (d'où le thème dérivé de la " trahison des clercs "), soit dans celle d'un refuge contre toutes les pressions de l'environnement extérieur. En bref, l'EDM, c'est tout ce qui s'oppose à l'appropriation collective des outils de travail et des modes de vie. On touche ici aux éléments centraux de la recomposition entre le service public et l'Europe. La fonction sociale du service public L'explication que l'on peut donner de la crise de 1995 relève d'un décalage croissant entre le mode de fonctionnement des services publics et leur fonction sociale. La construction européenne ne s'identifie pas à l'EDM et la déréglementation des services publics qu'elle entraîne doit être comprise comme une réponse à la libéralisation du marché des flux de biens et de services mise en œuvre par l'Organisation mondiale du Commerce. La privatisation progressive de France Télécom, l'ouverture des lignes 
aériennes intérieures au " cabotage " des compagnies étrangères, la séparation envisagée entre la fourniture de transports ferroviaires et l'entretien du réseau (à l'instar de ce qui s'est produit en Grande-Bretagne ou en Allemagne) s'inscrivent dans le jeu de plusieurs logiques cumulatives qui ne vont pas toutes dans la direction de la fin des monopoles :

- la logique économique de la concentration industrielle, à un moment où les gains de productivité offerts par les technologies nouvelles permettent de faire des économies d'échelle importantes et donc de rendre particulièrement rentables les grands marchés ; ces mécanismes de concentration ne peuvent que produire des réactions en chaîne, à partir du moment où une proportion importante d'un marché a été déréglementée (c'est le cas du transport aérien, déréglementé aux Etats-Unis en 1979, sous une présidence démocrate) ;

- la logique culturelle de la demande sociale, celle-ci s'étant considérablement diversifiée tant en matière de transports que de biens et de services culturels; à ce titre, la déréglementation n'est pas que le produit de pressions corporatives mais constitue aussi une réponse à la fragmentation d'une demande qui ne se satisfait plus de produits standardisés et à une culture consumériste où se jouent toujours les distinctions sociales; à la modulation des tarifs suivant les catégories de clients (industriels ou particuliers) s'adjoint la nécessité d'élargir la gamme des produits disponibles (en témoigne la politique commerciale de France Télécom) ;

- la logique politique, enfin, puisque à la déréglementation des services en réseaux qui correspond à la fin des monopoles naturels se conjuguent depuis le début des années 1980 de nombreuses réglementations nouvelles visant à protéger les consommateurs, la santé publique ou l'environnement; cet équilibre a d'ailleurs permis l'alliance des dérégulateurs et d'un Congrès démocrate aux Etats-Unis. Cette évolution montre que la déréglementation reste un enjeu politique dont l'issue est davantage déterminée par le rapport de force en faveur des groupes d'intérêt public que par l'application mécanique d'une logique marchande. Le tournant de la déréglementation ne peut être le même pour tous les services publics en France car leur positionnement à l'égard de ces trois logiques peut être très différent. Si Air France a pris tant de retard sur British Airways dans l'évolution de sa stratégie, c'est que la compagnie nationale a servi pendant longtemps des objectifs politiques et culturels (assurer la présence de la France sur des lignes déficitaires) et que la diversification des services s'est heurtée à une tradition " maison " héritée des pionniers de l'aviation et reposant pour l'essentiel sur la figure mythique du pilote au détriment du savoir-faire commercial. Si les ingénieurs de France Télécom ont largement soutenu l'idée de la déréglementation en Europe, c'est que l'entreprise disposait d'un savoir-faire technologique considérable et de solides parts de marché dans le monde entier. La logique économique leur était favorable tout comme l'environnement politique puisque les télécommunications permettaient des regroupements industriels dans le cadre d'une politique volontariste. Appropriée par l'un des grands corps techniques les plus mobiles (les X-Télécom), et dont les membres sont très présents dans le secteur industriel privé, la politique des télécommunications a permis autant de jouer sur la Commission pour faire avancer le dossier de la déréglementation que de désigner les opérateurs concurrents qui allaient intervenir à partir de la libéralisation totale des services en $1998^{11}$. La situation de la SNCF est très différente puisque l'impératif économique lui a totalement échappé et que l'évolution culturelle, favorisant l'automobile, lui est devenue hostile. La SNCF n'a jamais pu définir la politique de transports ferroviaires mais a été mise systématiquement au 
service de la politique d'aménagement du territoire dont le dernier avatar a consisté à multiplier des lignes de TGV non rentables. De nombreux surcoûts lui ont été imposés aussi par le développement d'une contestation politique des élus ou des groupes de défense de l'environnement. On pourrait certainement multiplier les exemples : EDF partage bien des points communs avec la SNCF (politique électronucléaire imposée par le gouvernement afin de garantir l'indépendance nationale et contestation politique forte de ses implantations dans un sens ou dans l'autre) mais s'en distingue néanmoins par la maîtrise de technologies de pointe et sa capacité forte d'exportation à des prix très compétitifs. En bref, deux variables communes semblent peser sur la capacité interne des services publics de négocier la déréglementation contrôlée de Bruxelles :

- d'une part, le fait qu'une entreprise publique détienne ou non une position d'acteur principal dans la définition de la politique publique qui la concerne; c'est le cas de France Télécom ou d'EDF mais pas de la SNCF ou d'Air France; la question se résume parfois dans le point de savoir si la demande politique et commerciale peut être sinon contrôlée, du moins maîtrisée. La question de l'indépendance des entreprises publiques à l'égard du pouvoir politique est posée depuis les années 1970. Elle cache néanmoins souvent la nature de l'interdépendance, celle-ci pouvant déboucher sur la colonisation des ministères de tutelle du fait de la densité des réseaux corporatifs (les X-Télécom aux PTT, les X-Mines aux Transports ou à l'Industrie).

- d'autre part, la sociologie interne de ces entreprises, qui dépend pour beaucoup de l'existence d'un groupe central, chargé de structurer en interne l'évolution de l'entreprise, et de la nature des relations qu'il entretient avec l'ensemble des salariés. La crise de 1995 ne s'éclaire que si l'on prend en considération le fait que la plupart des grandes entreprises publiques ont engagé une mutation " managériale " depuis des années. Elle s'inscrit dans une longue suite de conflits qui ont parfois mis en évidence la dualisation des entreprises publiques entre un état-major acquis aux objectifs de la déréglementation et un personnel inquiet pour ses conditions de travail. Lors de la crise d'Air France en 1993, la direction mise en place par Bernard Attali fut directement mise en cause par les grévistes pour " ne jamais aller voir comment se passaient les choses sur les pistes ". Dans ce processus de recomposition, où les acteurs cherchent leurs marques (y compris des syndicats faibles et mal organisés), l'Europe sert clairement de catalyseur, c'est-à-dire de facteur accélérant d'un processus déjà à l'œuvre $^{12}$. La question n'est donc pas celle d'une confrontation brutale entre un projet libéral et des monopoles publics. L'action européenne n'est pas en effet récente et ne date pas de 1995. La Cour de Justice décide dès 1964 que l'électricité est une marchandise et la première directive générale prise sur la base de l'article $90 \$ 2^{13}$, concernant la transparence des relations financières entre les Etats et les entreprises publiques date de 1980. Depuis 1988, se sont succédées, sans conflit particulier, ${ }^{14}$ de nombreuses directives concernant l'ensemble des services en réseaux alors que les opérations de privatisation se multiplient à vive allure en Grande-Bretagne (privatisation des chemins de fer en 1994) ou en Allemagne (séparation la même année des Postes et Télécommunications en trois entités distinctes, Deutsche Telekom étant privatisée et introduite en Bourse en novembre 1996). L'activation par la Commission comme par la Cour de Justice de l'article 90 n'est réellement opérée qu'au moment où des demandes convergentes proviennent des Etats membres pour que la libéralisation des services en réseaux soit organisée et contrôlée. En fait, l'action européenne vient entériner et accompagner un processus international de déréglementation puissamment relayé par la révolution thatchérienne et la recherche par tous les Etats 
de gisements d'économies budgétaires. La déréglementation ne se réduit pas à transmettre mécaniquement des décisions prises outre-Atlantique. Elle devient aussi un moyen de dégager les gouvernements nationaux de la gestion désormais bien risquée de l'Etat-providence. Un des objectifs principaux est de dégager des marges de manœuvre financières mais aussi de faire porter le poids politique des décisions tarifaires soit aux entreprises publiques elles-mêmes soit aux collectivités locales. Ce transfert politique n'a pu être effectué en France étant donné l'intégration des élites et l'unicité du pouvoir d'Etat. Les alternances, favorisant les nominations politiques à la tête des grandes entreprises, n'ont pu que brouiller les frontières entre ce qui relevait de la décision centrale et ce qui devait relever de la gestion des services publics. Mais cette mutation, souvent très sensible pour les personnels, est restée englobée dans les projets politiques plus généraux visant à construire l'Europe. En France, le " Plan Juppé ", présenté comme une conséquence de l'EDM, devient un nouveau discours de la contrainte financière pour des entreprises publiques qui ont subi déjà de nombreuses mutations depuis la fin des années 1980. A la différence des pays étrangers, cependant, ces mutations, souvent présentées comme le fleuron des pratiques gestionnaires publiques, ont été mal absorbées. D'une part, la réforme des entreprises publiques n'est pas associée en France, comme elle l'est en Allemagne, en Grande-Bretagne ou en Italie, à une réforme plus générale de l'Etat consistant à réduire les charges publiques, à systématiser l'emploi de techniques managériales dans les services centraux ou à redéployer les compétences de gestion entre le centre et la périphérie: si le cadre fédéral permet aux Länder allemands de prendre en charge la gestion des réseaux ferroviaires, la régionalisation de la SNCF lancée à titre expérimental pour le trafic voyageurs en 1997 ne peut être que partielle et soumise à des conditions restrictives, les régions étant particulièrement pauvres en services de gestion. En bref, les entreprises publiques en France doivent subir une mutation bien plus importante que les ministères ou les administrations de gestion, assez largement bloqués par la hiérarchie des corps et la différenciation sociale des pratiques professionnelles, alors même que le cadre normatif général ne se prête guère à cette mutation. D'autre part, les réformes opérées dans les entreprises publiques, afin de développer de nouvelles pratiques gestionnaires, ne s'intègrent pas dans des projets institutionnels cohérents. Le personnel de France-Télécom, après avoir célébré le maintien du statut de fonctionnaire pour les agents en place (les recrutements de fonctionnaires perdurant jusqu'en 2001), ne sait pas comment la cohabitation se fera avec les nouveaux arrivants recrutés sur contrats de droit privé. L'organisme, qui se perçoit et s'énonce lui-même comme une entreprise, opère de plus de nombreux changements d'affectation impliquant des formations aux fonctions commerciales. Les congés de fin de carrière, négociés avec les syndicats, associent le changement de structure au changement de génération. A La Poste, la réduction des effectifs fonctionnaires (passés de 276000 à 254 000 entre 1991 et 1996) s'est traduite par une charge accrue de travail mais aussi par l'augmentation sensible de la proportion d'agents recrutés sur contrats de droit privé qui compte pour un sixième de l'ensemble du personnel. Là encore, le maintien d'une structure d'exploitant public a pu apparaître formelle étant donné la définition d'une stratégie d'entreprise clairement énoncée en termes de chiffre d'affaire et la multiplication de procédures permettant d'assouplir le tradition fonctionnement de l'organisme. Par ailleurs, le développement des techniques de gestion a conduit à développer des structures beaucoup plus autonomes et diversifiées venant contredire une centralisatrice bien établie, sur laquelle les grands syndicats s'étaient d'ailleurs 
modelés. Les critères de la réussite professionnelle ont eux-mêmes changé y compris dans les secteurs les plus " techniques ", comme EDF, où les notions de " service " et de suivi de la clientèle sont entrées en force. Aux carrières faites à l'ancienneté, permettant de gravir les échelons hiérarchiques successifs, ont succédé un blocage de l'avancement, dû au raccourcissement de la ligne hiérarchique et à la réduction des effectifs, et une stratification plus forte entre les états-majors et les services opérationnels. Les entreprises publiques françaises ont ainsi progressivement perdu ce qui faisait leur originalité sociale, à savoir la possibilité qu'elles offraient d'une promotion sociale. Bien plus, la plupart d'entre elles (notamment la SNCF et l'EDF) s'inscrivaient dans des traditions professionnelles (les " cheminots ", les " électriciens ") qui correspondaient à une évolution historique liée aussi bien aux événements de la guerre qu'aux nationalisations de l'après-guerre. Cette tradition était faite de mémoire collective et de stratégies sociales bien repérées puisque l'entreprise correspondait à un secteur de la modernisation économique mais aussi à un mode de vie. A cela s'ajoutait une forte hérédité socio-professionnelle qui faisait que l'on pouvait être cheminot de père en fils. La plupart des revendications apparues durant le conflit de 1995 ont d'ailleurs été associées au refus de voir disparaître cette norme collective, les grévistes soulignant à l'envi qu'il fallait que leurs enfants puissent bénéficier des mêmes conditions de travail. Enfin, un troisième élément paraitt propre à la conception du service public qui a prévalu en France depuis les années 1980. Depuis les nationalisations de l'après-guerre, les entreprises publiques ont été généralement utilisées pour mettre en œuvre des politiques étrangères à leur " objet ". Si EDF, la SNCF ou France-Télécom ont été associées aux politiques industrielles dans les années $1960^{15}$, ces entreprises ont été chargées de politiques sociales dans les années 1980, qu'il s'agisse, bien entendu, du maintien des péréquations tarifaires ou de l'engagement dans des opérations de lutte contre l'exclusion. C'est ainsi qu'EDF-GDF s'est associée depuis 1985 aux départements dans le cadre de conventions précarité-solidarité pour la prise en charge totale ou partielle des factures impayées, soit un coût de 28 millions de francs en 1995 pour la seule entreprise. En 1996, une charte a été signée entre le ministère du Logement, EDF-GDF et le syndicat des distributeurs d'eau afin que les usagers en situation précaire puissent néanmoins bénéficier d'une fourniture d'énergie. L'ensemble de ce mécanisme doit être financé par l'impôt. Alors que La Poste génère un déficit d'un milliard de francs par an et que $65 \%$ de ses recettes proviennent de ses activités financières, il lui a fallu maintenir un réseau rural de 17000 points de contacts créé au siècle dernier afin de maintenir une présence territoriale. Du fait de l'urbanisation, $60 \%$ de ces bureaux ne touchent que $20 \%$ des usagers, ce qui entraîne au passage une inégalité croissante entre le service des villes et le service des campagnes : un bureau pour 900 habitants en Lozère contre 15000 habitants en Seine-Saint-Denis. La notion de " mission particulière " des entreprises publiques, utilisée par l'article $90 \$ 2 \mathrm{CE}$, est donc loin d'être univoque. La diversification des missions, la superposition des enjeux externes (accroître la clientèle) et internes (mobiliser les salariés autour d'un projet cohérent), la multiplication des registres de légitimation ont créé de fait une situation d'anomie chez les salariés de ces entreprises qui ne disposent plus d'une norme professionnelle qui leur permette à la fois de définir leur métier et, très concrètement, de jouer le jeu de l'évaluation managériale ${ }^{16}$. Le service public " à la française " résulte ainsi d'une concrétion de buts et de moyens que la déréglementation fait éclater. On passe ainsi du service public comme institution à la notion de service public comme fonction. Le service public: de l'institution à la fonction L'intégration 
européenne a pour effet d'éclairer les pratiques nationales en matière d'aides publiques, de mieux connaître les coûts réels des activités publiques et à clarifier les rapports entre l'Etat et les entreprises publiques. Si la Commission et la DG IV ont pu être parfois instrumentalisées par les Etats membres, afin de contourner les résistances syndicales ou politiques nationales à la déréglementation, une nouvelle étape a été franchie dans la seconde moitié des années 1990 lorsqu'une régulation européenne des grands services en réseaux est devenue nécessaire. On est alors passé d'une accumulation de mesures partielles à l'étape institutionnelle appelant la définition d'une véritable politique réglementaire qui fasse la part de l'économique et du social. Certaines questions doivent trouver réponse car les Etats membres, et notamment la France, ne pourront longtemps se satisfaire de solutions provisoires (comme le gel de la réforme de la SNCF décidé en novembre 1996), c'est-à-dire d'une semidéréglementation sans adaptation des structures. De telles solutions se chargent désormais de risques considérables, qu'ils soient politiques (des grèves impopulaires à répétition) ou bien économiques (ne pas pouvoir s'opposer à la concurrence internationale qui vient " écrémer " les services les plus rentables). Une de ces questions concerne le point de savoir si les instances de l'Union peuvent définir une politique du service public. En quoi, notamment, les concepts de service économique d'intérêt général et de service universel peuvent-ils suppléer l'absence d'un concept de service public presque introuvable ${ }^{17}$ dans les traités? La notion de service d'intérêt économique général est utilisée par la Cour de Justice, à l'issue d'une jurisprudence abondante, afin de circonscrire le champ d'application de l'article 90\$2. Cette jurisprudence pose deux normes fondamentales : la première, c'est que l'appréciation de la mission d'intérêt général reste du domaine des autorités publiques nationales, sous le contrôle de la Cour ; la seconde, c'est que le critère principal de jugement reste l'effet sur le marché. C'est ainsi que les règles de la concurrence ne peuvent être écartées que si elles interdisent la poursuite de la mission d'intérêt général ou compromettent la viabilité économique de l'entreprise. La combinaison des articles $90 \$ 2$ et de l'article 86 s'opposant aux subventions croisées lorsqu'elles sont prélevées sur des clients captifs, et donc aux péréquations tarifaires, conduit à ne réserver les exceptions au principe de concurrence qu'aux activités relevant du service universel ${ }^{18}$. Le concept de service universel est-il alors synonyme de service public ? Cette notion a été développée aux Etats-Unis à la fin du XIXe siècle pour justifier la création d'un monopole téléphonique par ATT qui désirait opérer l'interconnexion de tous les réseaux. L'Europe a repris le terme en lui adjoignant une connotation sociale. L'expression apparaît pour la première fois en 1987 dans le Livre Vert sur la libéralisation des télécommunications, puis se trouve esquissée en 1992 dans un rapport de la Commission sur la situation du secteur des télécommunications. Le service universel est alors conçu comme un réseau doté d'une couverture géographique générale, accessible à tout usager sur sa demande dans un délai raisonnable et à des prix abordables. La notion est là encore de nature stratégique et matérielle: la libéralisation totale des télécommunications est compensée par la garantie d'un service minimum. Dans une déclaration du 16 février 1994, la Commission généralise le concept de service universel sur la base de trois normes: l'universalité (accès de tous à des conditions abordables), l'égalité (couverture géographique générale), la continuité (service permanent à un niveau de qualité défini). La similarité de ces principes avec les trois " lois de Rolland " concernant le service public à la française ne doit pas faire illusion. Car la notion de service universel ne concerne que la prestation fournie aux 
usagers, sans que celle-ci implique une organisation particulière du service. L'article $222 \mathrm{du}$ traité de Rome confirme du reste que l'Europe reste étrangère au régime de propriété dans les Etats membres. La notion de service universel renvoie seulement à la qualité minimum qu'un usager individuel est en droit d'attendre d'un réseau téléphonique ou postal moderne en Europe. De plus, l'exclusion des règles de la concurrence ne touche pas l'ensemble du service universel mais les seuls " services réservés " qui peuvent justifier les monopoles et les péréquations. La question reste ouverte quant à savoir quelle part des services universels échappe aux services réservés et peut donc, en fonction de la technologie mais encore des pratiques de consommation, être ouverte à la concurrence. L'intervention européenne conduit donc à séparer la fonction de l'organisation. Le service public à la française ne se distingue pas par des critères de qualité économique ou par un système de financement public. Il constitue une institution au sens où l'intégration des fonctions de service dans des organisations sous contrôle public forme un des éléments de l'ordre politique. La qualité publique ou privée de ces organisations comme les statuts des personnels ne sont pas à prendre en considération car l'institution dépasse la logique juridique formelle ${ }^{19}$. La superposition de politiques industrielles et sociales même contradictoires impose au service public à la française un travail de nature politique, fait de compromis entre groupes antagonistes et intérêts divergents de part et d'autre des frontières de l'organisation. Les entreprises publiques en France ont permis l'insertion de corporatismes professionnels dans le système politique en leur confiant une fonction d'intérêt général, tout comme la gestion des intérêts patrimoniaux et des successions à été confiée aux notaires ou la santé des familles aux médecins libéraux. Le service public en France n'est donc pas une juxtaposition de fonctions et d'organisations aux règles un peu désuètes. C'est une institution, c'est-à-dire, pour reprendre les termes d'Hauriou, une idée œuvre qui devient porteuse d'une norme sociale ${ }^{20}$. On ne peut donc réduire le débat sur le service public à la qualité du produit ou au " service du public " que la déréglementation permettrait enfin d'obtenir. L'idée qui prévaut dans le service public c'est qu'il existe un échange politique qui échappe aux règles du marché économique, l'usager n'étant pas nécessairement un client pas plus que l'électeur n'est nécessairement un stratège. Derrière le service public réside donc une notion centrale de volontarisme politique auquel semble s'opposer une logique européenne de type conséquentialiste. En effet, les autorités de l'Union s'appuient sur les principes élémentaires de la cohésion sociale (égalité d'accès, prix raisonnable) pour définir ce qu'est un service universel sans s'interroger sur la notion elle-même. L'Europe entérine une séparation des fins et des moyens alors que l'institution formalise au contraire leur relation. A ce titre, on ne doit pas sous-estimer le fait que l'Europe est aussi porteuse d'un modèle institutionnel bien particulier qui se traduit notamment par la séparation des opérateurs de services publics et des régulateurs, qu'il s'agisse d'agences nationales ou bien d'instances juridictionnelles (ou bien encore d'un mélange des deux dans le cadre de commissions régulatrices à l'image de certaines autorités administratives indépendantes françaises ou des regulatory commissions américaines) ${ }^{21}$. Là encore, on voit apparaître une spécification qui impose de donner une organisation particulière à chaque fonction. Mais on sait aussi que la notion de régulation ne trouve pas à s'insérer en France dans un cadre institutionnel adapté. Les autorités administratives indépendantes ont souvent été capturées par les administrations de gestion, n'ont pas toujours échappé au processus de politisation et n'ont pas constitué un modèle administratif alternatif crédible. Cet enjeu institutionnel montre que toutes les 
fonctions ne peuvent pas se développer dans un système politique sans support adéquat, c'est-à-dire sans formules d'organisation qui fassent la part des hiérarchies sociales et des nécessités du contrôle démocratique ${ }^{22}$. Le concept de régulation renvoie notamment à la place que l'on entend donner à l'expertise dans un schéma de pouvoir. C'est d'ailleurs là que l'on trouve l'une des limites principales à la réforme de l'Etat en France. Le passage à un Etat régulateur suppose en effet de remettre en cause le primat social et culturel de la décision dans l'univers de la classe politique. Au-delà des difficultés propres à la technologie administrative, les réformateurs se heurtent aux normes professionnelles qui font de l'expertise un instrument secondaire de pouvoir comme en témoigne l'échec de l'évaluation des politiques publiques. La crise de 1995 est donc à placer au rang des divers signes montrant que l'Europe, pour les leaders politiques nationaux, reste difficile à expliciter, jouant sur des registres conceptuels étrangers à la culture politique française ${ }^{23}$. On serait alors tenté de dire que l'Europe reste toujours la figure de l'anti-politique, mais une telle interprétation conduit à rejoindre la critique sans nuance de l'EDM ou bien à faire la constatation somme toute banale qu'il n'existe toujours pas de gouvernement européen chargé de rationaliser et d'expliciter les référents de l'action communautaire, tâches qui sont pour l'instant dévolues à la Cour de Justice. Une autre voie intellectuelle consiste plutôt à s'interroger sur le sens de ces innovations conceptuelles ${ }^{24}$ : l'Europe serait-elle la figure de proue d'une pouvoir politique postmoderne? Pourrait-on voir dans la dichotomie entre les fonctions et les organisations un modèle nouveau de pouvoir libéré des anciens principes de légitimation, une " gouvernance sans gouvernement " ? Il faut en effet considérer que la définition historique des institutions comme le service public a répondu à la nécessité d'associer des compromis sociaux à la satisfaction de besoins économiques. Le pouvoir politique de l'après-guerre a trouvé une puissante légitimation dans cette capacité d'organiser ainsi la vie sociale suivant des formules originales (l'entreprise publique, par exemple) au-delà des réquisits de la démocratie électorale. En jouant la carte du management public, qui offre toute une palette de solutions plus ou moins souples pour distinguer ce qui relève du pilotage et ce qui relève de la mise en œuvre, le pouvoir, et plus particulièrement le pouvoir d'Etat, n'aurait plus à fournir de modèle institutionnel. Pour reprendre l'argument postmoderne, l'Europe permettrait d'entériner la multiplication de configurations socio-professionnelles (les services en réseaux, les services régaliens maintenus dans le giron de la subsidiarité nationale, les services locaux, etc.) qui n'auraient aucune vocation à s'intégrer dans un modèle global. L'Europe fournirait alors l'exemple d'une pragmatique, dégagée de tout récit à prétention universaliste (la généralisation du mode de vie occidental, l'émancipation des travailleurs, la doctrine des Lumières et tutti quanti) qui trouverait à se déployer dans des instruments contractuels, scellant des accords momentanés entre des acteurs placés dans une situation de justification équivalente : l'usager, devenant consommateur à part entière de services collectifs, n'aurait pas à payer le surcoût dû aux situations de monopoles, ou aux arrangements passés entre le pouvoir et les syndicats au nom de la paix sociale. Les relations de classes, analysées en termes marxistes par la critique de l'EDM, feraient alors place, dans cette hypothèse, à des relations de groupes en concurrence pour l'accès à la définition des champs légitimes de l'action collective. Le déterminisme local remplacerait l'institution ${ }^{25}$. Malheureusement, cette hypothèse postmoderne se heurte à l'affirmation claire d'un nouveau méta-récit, celui de l'efficacité et de l'enrichissement collectif, tel qu'il est porté par l'Europe depuis le traité de Rome. On 
est loin d'une situation où des groupes organiseraient l'action publique en fonction d'un dialogue argumenté entre des dimensions " incommensurables " (la qualité du service contre la socialité offerte par le service public). Bien au contraire, puisque la séparation des moyens et des fins réduit à peu de chose le contrôle social que les groupes peuvent espérer obtenir sur le produit final de l'action publique. C'est bien ce que l'on trouve, du reste, au cœur de la contestation de 1995 : un aveu d'impuissance face à la nécessité désincarnée de respecter les règles de l'économie financière. Bien plus, cette crise a donné la preuve empirique que le débat conceptuel n'opposait pas une modernité d'Etat bureaucratique et vermoulue aux partisans d'une postmodernité faite de réseaux souples et autopoïétiques. L'institution a été défendue par les groupes hors-système du syndicalisme et l'acquiescement d'une partie de l'opinion alors que les représentants de l'Etat endossaient maladroitement le rôle de réformateurs ultralibéraux. Un autre effet de l'intégration européenne est donc de pousser à s'interroger sur la nature des institutions politiques nationales. On ne peut pas attendre de l'Union qu'elle résolve par elle-même des équations que les Etats membres ont toujours refusées ou ont été incapables de réaliser. Il ne faut donc pas inscrire la construction de l'Union européenne dans le seul cadre d'une logique libérale venant contredire une tradition d'interventionnisme public fort. Il faut aussi s'interroger sur les instruments et les outils conceptuels qui permettront à la France de négocier la déréglementation et de concilier les demandes de l'Union avec ses propres structures politiques. On est ici confronté à des seuils qualitatifs et non pas à des réalités dégradables, sauf à utiliser des concepts à géométrie variable. Le débat récent sur la notion de service d'intérêt général peut en témoigner. La crise de 1995 en France a suscité de nombreuses prises de position devant toutes échouer pour avoir voulu inscrire la notion de service public dans les textes, qu'il s'agisse de la Constitution de la VeRépublique ou du traité de Rome. Alors que le rapport public de 1994 du Conseil d'Etat ${ }^{26}$ défendait l'idée de service public comme élément de souveraineté nationale, et donc l'associait aux éléments constitutifs de l'espace politique, le rapport Denoix de Saint-Marc ${ }^{27}$ entérinait en 1996 la séparation des missions de service public de l'organisation en monopoles publics tout en maintenant contre vents et marées l'idée que " ...notre pays doit contribuer à l'élaboration d'une doctrine européenne du service public, sous ce nom ou sous un autre. " (c'est nous qui soulignons). Le rapport précise que cette notion de service public doit reposer sur des " ...principes partagés dans tous les Etats membres et sur des objectifs d'intérêt général européen: le dynamisme des marchés et la défense des intérêts des consommateurs, mais aussi la garantie des libertés et droits fondamentaux, en particulier dans le domaine social, ainsi que la sauvegarde d'intérêts communautaires... ". En ajoutant que ces principes constituent les fondements du service public, le rapport de 1996 ne fait que reprendre les arguments développés par la jurisprudence de la Cour de Justice pour illustrer le service universel. En d'autres termes, la notion de service public dégagée de son contexte politique devient inopposable au niveau communautaire sauf à décrire ce que l'Union a déjà élaboré. Dans ce contexte, on peut s'interroger sur le point de savoir si la reconnaissance par la Commission dans sa communication du 11 septembre 1996 de la notion de services d'intérêt général constitue une véritable innovation. Il semble que la Commission est restée très fidèle à la doctrine communautaire en distinguant ceux-ci des services d'intérêt économique général par le fait qu'ils désignent des services non- marchands. Le cadre d'interprétation reste néanmoins toujours le même : " La solidarité et l'égalité de traitement dans le contexte d'une économie de marché ouvert et dynamique 
constituent des objectifs fondamentaux de la Communauté européenne. Les services d'intérêt général contribuent à la réalisation de ces objectifs. Les Européens attendent des services de qualité à un prix abordable " ${ }^{28}$. Le fait que ces services soient considérés comme étant " au coeur du modèle européen de société " ne l'empêche pas de bien distinguer dans les deux paragraphes suivants les missions des services d'intérêt général. Bien plus, le contenu des services d'intérêt général est laissé aux bons soins des Etats membres au nom de la subsidiarité ( $(18)$ et la mise en œuvre de ceux-ci reste toujours conditionnée par le principe de proportionnalité (§21). L'avenir du service public dépendra, selon toute probabilité, de l'équilibre réalisé entre le principe de subsidiarité et la concurrence désormais ouverte pour trouver les meilleures formules d'organisation. Dans ces conditions, même le service universel sera plus le résultat d'un ensemble d'agencements économiques et sociaux modulables et révisables en permanence ${ }^{29}$ qu'un objet social défini par l'histoire politique. Le service public, perdant sa qualité d'institution, devient le produit ou l'effet d'arrangements négociés qui devraient s'étendre, en toute logique, aux relations professionnelles ${ }^{30}$. Conclusion L'apport conceptuel de l'Europe, au détour du débat sur le service public en France, pourrait donc se présenter ainsi :

- la fin du service public comme institution indique que l'Europe joue un rôle catalyseur dans l'évolution socio-politique de la France. L'institution du service public était associée à l'idée d'un espace public où s'effectuait le bien alors que la séparation des moyens et des fins opérée par l'Union désigne un espace public tendu vers le juste ou l'équitable. Une telle visée théorique se traduit par la recherche d'une spécification des organisations et des politiques, par la recherche d'une plus grande transparence des financements. Cette idée d'équité trouve de puissants échos dans l'évolution de la pensée politique en France où elle est reprise pour justifier la mutation des systèmes d'assurance sociale, le renforcement du pouvoir judiciaire ainsi que les divers éléments d'une conversion au libéralisme. L'Europe comme catalyseur, donc, et non comme variable externe agissant de manière mécanique sur les systèmes socio-politiques nationaux. L'Europe ne peut être appréhendée dans ce débat ni comme contrainte externe ni comme instrument d'une manipulation opérée par les élites nationales (qui ne sont pas toutes, loin s'en faut, converties au libéralisme!), ces deux interprétations faisant la part belle au mythe de l'EDM ;

- l'Europe n'est pas a priori une machine à produire des concepts politiques mais des concepts juridiques devant permettre la réglementation de secteurs d'activité économique. Néanmoins, la mise en place du grand marché tend à créer des éléments de politique institutionnelle qui peuvent venir contrarier, voire transformer les normes socio-politiques nationales. Cette transformation peut s'étendre jusqu'aux normes fondatrices du pouvoir politique, lui interdisant d'utiliser des registres de légitimation qui lui étaient jusque là coutumiers (l'appel au " service public "). A ce titre, les notions d'apprentissage, d'adaptation, de convergence, ne peuvent guère être utilisées ici car elles éludent la question du changement... ou du conflit. Le passage d'un modèle institutionnel à un autre se fait par seuils qualitatifs, ce qui explique la crise des entreprises publiques à statut en France et, plus largement, les difficultés d'entériner une forme radicale de réforme de l'Etat. Ce qui est en jeu ne se réduit pas en effet à " moderniser " les entreprises publiques mais à remettre en cause la spécificité du secteur public tout entier. Le registre de l'" apprentissage " ne fait que reprendre l'énoncé libéral de la théorie systémique qui suppose toujours une adaptation des systèmes à l'environnement sans trop se préoccuper de ce que sont les boîtes noires, 
c'est-à-dire, très concrètement, des populations et des structures de pouvoir qui doivent appréhender ce changement. C'est le discours typique des consultants en management qui savent aussi quel est le prix à payer pour ceux qui n'apprennent pas assez vite, et qui n'ont guère le choix entre le voice et l'exit. On retrouve, du reste, cette litote de l'apprentissage dans le discours tenu par le gouvernement durant tout le conflit de 1995 : il suffit de bien expliquer, de " communiquer ", de se " concerter " pour qu'une négociation soit inutile et que la réforme soit entérinée ;

- la séparation de la régulation et de la gestion, de l'administratif et du politique participent d'un nouveau modèle institutionnel qui se développe dans les divers programmes de réforme de l'Etat. La construction communautaire vient à la rencontre d'une redéfinition nationale (en France mais aussi dans d'autres Etats membres) du pouvoir politique. Celui-ci est alors débarrassé des normes collectives échappant à l'artificialisme, c'est-à-dire de normes pouvant témoigner d'un ordre extra-contractuel, qui ne se traduise pas par des bilans coûts/avantages. Ici s'opère sans doute tout un jeu complexe derrière le passage à la " gouvernance ", fausse théorie politique prônant le pouvoir sans domination et faisant accroire que les procédures contractuelles, renouant ainsi avec un solide juridisme, peuvent circonscrire, voire même contrôler les rapports sociaux. Le passage à la gouvernance, comme conversion à l'artificialisme absolu (et au relativisme des légitimations), et comme résultante d'un enchevêtrement de partenariats et d'accords provisoires, peut être aussi bien interprété comme l'accès à une forme de cité idéale, et l'on rejoint alors les utopies, que comme un moyen de neutraliser toute tentative de constituer une " centralité " politique permettant l'agrégation des intérêts. Car, quel que soit le succès des manifestations de 1995 contre le " plan Juppé ", il n'en demeure pas moins vrai que les grands services publics sont appelés désormais à s'engager dans une déréglementation généralisée et dans une nouvelle architecture du pouvoir qui nie la spécificité sociale du secteur public. Les théories de la postmodernité sont alors retournées contre leurs fins d'émancipation. En ce sens, l'Europe libère le pouvoir politique, mais peut-on s'en réjouir?

\section{NOTES}

1. Chargé de recherche au CEVIPOF/CNRS.

2. On reste ainsi sidéré par le fait que sur les quelques vingt ou vingt-cinq commentateurs autorisés de la crise de novembre-décembre 1995 un seul, à notre connaissance, se soit interrogé sur l'évolution interne des services publics en France et la dimension organisationnelle du conflit (Jean-Pierre Le Goff, " Le grand malentendu " in Jean-Pierre Le Goff et Alain Caillé, Le tournant de décembre, La Découverte, Paris, 1996).

3. Sur cette dernière source d'incertitude, voir la mise au point d'Olivier Mongin et de Joël Roman, " L'électrochoc de novembre-décembre 1995 : premiers diagnostics ", Esprit, juin 1996, p. 185.

4. Pour une présentation critique des transformations du système politique français liées à la " gouvernance ", voir Jean Leca, " La gouvernance de la France sous la Ve 
République " in François d'Arcy, Luc Rouban (dir.), De la Ve République à l'Europe Hommage à Jean-Louis Quermonne, Presses de Sciences Po., Paris, 1996, p. 329 et s. 5. On peut citer en particulier la privatisation de 75 entreprises publiques en GrandeBretagne ou l'alignement du système de retraite de la fonction publique sur le droit commun en Italie.

6. Alain Touraine parle lui-même de " l'ombre d'un mouvement " in Alain Touraine et al., Le grand refus, réflexions sur la grève de 1995, Fayard, Paris, 1995.

7. Voir, en particulier, les commentaires d'Olivier Duhamel et Philippe Méchet, " Une grève d'opinion ", dans SOFRES, Etat de l'opinion 1996, Seuil, Paris, 1997, p. 33 et s.

8. " Ainsi la gauche, au lieu d'incarner l'autonomie de la société, s'est installée dans le même univers, dans une même représentation du monde que son adversaire historique : la domination absolue sur la nature, la maîtrise rationnelle du monde et de la " productibilité " illimitée de toute chose. En donnant la priorité à l'économique sur le social, elle a légitimé un pouvoir extra social, celui des forces économiques dominantes bloquant ainsi la capacité de critique et de réflexion de la société, délégitimant les luttes ou mobilisations sociales potentiellement porteuses d'émancipation ou de libération, craignant en fait toute radicalisation d'une société considérée comme une masse incohérente de désirs contradictoires ". Bernard Ravenel, " Un mouvement social politiquement orphelin ", M-Marxisme, mai-juin 1996, p. 10. 9. Comme le souligne Pierre Bauby, prenant néanmoins une certaine distance avec le discours sur l'EDM : " Cette crise de l'insertion de la France dans la mondialisation se double d'une crise de l'intégration européenne, du moins de la mise en cause de sa logique jusqu'ici dominante, particulièrement sensible à l'égard du processus de libéralisation des services publics... Mais, le plus souvent, l'argument anti-européen apparaît comme un alibi et l'Europe comme un bouc-émissaire facile pour éluder les responsabilités propres des politiques. Le traité de Maastricht n'oblige pas à avoir une politique monétaire aussi restrictive et ne dicte pas les processus internes de régulation sociétale. Il reste que l'Europe est souvent perçue comme le produit d'une pure volonté des élites, une construction abstraite à partir de l'économie et du droit, une dépossession de la souveraineté populaire... ", " L'unité contradictoire du mouvement social de novembre-décembre 1995 ", M-Marxisme, mai-juin 1996, p. 16.

10. Voir en particulier l'article de Pascal Perrineau et Michel Wieviorka, " De la nature du mouvement social ", Le Monde, 20 décembre 1995.

11. Elie Cohen montre bien d'ailleurs que France Télécom a préféré jouer la carte de la déréglementation des services plutôt que celle d'une séparation organique entre les activités locales et les activités internationales de crainte de perdre tout contrôle sur la politique des télécommunications : La tentation hexagonale, Fayard, Paris, 1996, p. 200. 12. Comme l'a remarqué très justement Jean-Louis Quermonne dans le commentaire qu'il a fait d'une première version de ce texte, et dont je tiens à le remercier, on peut aussi concevoir l'intervention des institutions européennes comme une médiation à l'image du travail accompli au lendemain de la guerre par le Commissariat général du Plan pour préparer la société française au choc des Trente Glorieuses. Je partage tout à fait cet argument, qui montre en réalité la capacité surprenante du système politicoadministratif français d'inventer, ou de contrôler, des moyens institutionnels nouveaux afin de préserver des équilibres sociaux fondamentaux (et des positions de pouvoir). Je conserve néanmoins ici le terme de " catalyse " pour évoquer le déplacement produit par l'intervention des instances communautaires sur le champ des concepts et des pratiques en matière de service public. Si le risque principal était constitué après 1945 
par une " américanisation " de la société française sur le modèle allemand, évolution souhaitée par certains commentateurs d'outre-Atlantique, le risque du prochain siècle serait plutôt celui d'un appauvrissement à l'image de ce qui s'est produit au Brésil ou au Mexique, lorsqu'ils furent brutalement confrontés à la déréglementation mondiale. 13. Qui étend le principe de libre concurrence aux entreprises publiques: " Les entreprises chargées de la gestion de services d'intérêt économique général ou présentant le caractère d'un monopole fiscal sont soumises aux règles du présent traité, notamment aux règles de concurrence, dans les limites où l'application de ces règles ne fait pas échec à l'accomplissement en droit ou en fait de la mission particulière qui leur a été impartie ".

14. C'est ainsi que la décision du Conseil de juin 1993 fixant la libéralisation totale des télécommunications pour 1998 est prise à l'unanimité.

15. Sur ce point, on peut se référer aux critiques émises par Elie Cohen : " La vraie spécificité française n'est donc pas dans l'invention du service d'intérêt économique général. Elle réside dans une conception du service public plus attentive au rayonnement de l'Etat social-colbertiste qu'au service du public ". La tentation hexagonale, op. cit., p. 186. On ne suivra cependant pas totalement l'auteur qui sousestime le poids des contraintes de politique sociale sur la définition des missions de service public.

16. Cette question ne se pose pas que pour les services publics industriels et commerciaux mais pour l'ensemble des services publics français, qu'ils soient purement administratifs ou sociaux. La notion d'évaluation est ainsi fortement contestée par les enseignants qui ne retrouvent pas dans les méthodes utilisées la réalité d'un travail en mutation.

17. La seule référence au service public apparait dans l'article 77 du traité de Rome et ne concerne que la politique des transports qui avait fait l'objet d'un investissement communautaire important à l'époque.

18. C'est une des conclusions que l'on peut tirer des commentaires que la doctrine a pu faire des deux arrêts de principe Paul Corbeau (CJCE 19 mai 1993) et Commune d'Almelo (CJCE 27 avril 1994). Dans les deux cas, c'est bien l'existence d'obligations de service universel qui autorisent et la péréquation tarifaire et une protection particulière contre des activités voisines ou annexes du service public mais qui pourraient mettre en cause la viabilité économique des entreprises publiques concernées. Sur ce point : Jean-Yves Chérot, " L'article 90, paragraphe 2, du traité de Rome et les entreprises de réseau ", Actualité juridique de droit administratif, 20 mars 1996, p. 171 et s.

19. En effet, la plupart des services publics locaux sont confiés à des entreprises privées travaillant dans le cadre de concessions. L'erreur de nombreux commentateurs de la crise de 1995 est d'avoir cristallisé le débat sur les statuts. La notion de service public, comme élément de la culture politique, ne respecte pas les critères juridiques. En témoigne l'identification réalisée par l'opinion (et les sondages) entre services publics marchands et non-marchands ou l'assimilation de La Poste, de la Sécurité sociale et de l'Education nationale dans un même univers de significations, malgré toutes les différences organisationnelles de ces secteurs.

20. La constitution du service public en France relève donc des politiques institutionnelles régulatrices au sens où l'entend Jean-Louis Quermonne, " Les politiques institutionnelles " in M. Grawitz et J. Leca (dir.), Traité de science politique, vol. IV, Paris, PUF, 1985, p. 79 et s. 
21. Il faudrait, une fois pour toutes, cesser d'opérer l'amalgame sémantique entre le concept français de régulation et le concept anglo-saxon de regulation qui renvoie à la notion bien connue de réglementation (celle-ci n'impliquant pas nécessairement une régulation, sauf à utiliser le schéma institutionnel américain). Le fait que les instances de l'Union produisent des réglementations ne signifie rien quant à leur capacité de réguler effectivement les économies ou les sociétés européennes. Cet amalgame sert en fait à justifier l'usage et l'extension des pratiques réglementaires de type accusatoire en vigueur aux Etats-Unis mais généralement sans tenir compte qu'il existe déjà aux EtatsUnis une structure politique fédérale et une hiérarchisation des agendas. Cette " exemplarité " américaine renoue avec les bonnes vieilles théories du développement politique. Une bonne illustration en est donnée par l'ouvrage de Giandomenico Majone, La Communauté européenne : un Etat régulateur, Montchrestien, coll. Clefs, Paris, 1996. 22. Par exemple, il ne sert à rien, comme le fait Giandomenico Majone, de prôner le modèle " quasi-judiciaire " des agences réglementaires aux Etats-Unis si l'on ne tient pas compte et de la culture et de l'histoire politique du pays, conférant un rang social particulier aux fonctions de type judiciaire. Dans le même ordre d'idées, finalement proches d'un fonctionnalisme normatif, on pourra relever que les représentants $\mathrm{du}$ libéralisme politique, tel François Léotard, évoquent clairement durant la campagne des législatives de 1997 la possibilité de changer le système constitutionnel français pour adopter un régime présidentiel à l'américaine, changement justifié en grande partie par l'intégration européenne et le rôle nouveau dévolu à l'Etat.

23. Il n'en va pas nécessairement de même pour les hauts fonctionnaires qui ont beaucoup à gagner dans le développement d'un Etat central " stratège ". Cette évolution viendrait en effet renforcer le pouvoir des grands corps et confirmer le rôle intellectuel que la haute fonction publique a toujours voulu assumer. Comme quoi l'espace ouvert par le passage à la " régulation " reste toujours un espace stratégique de positionnement social.

24. Que ces concepts institutionnels soient ou non bien maîtrisés par les acteurs dans le cadre des nombreux débats consacrés au service universel est une autre affaire. Les représentations socio-politiques sont bien plus souvent subies que construites par les acteurs (notamment certains députés français clamant leur attachement au " modèle français de service public " tout en défendant le principe d'une réforme managériale de l'Etat). Ces derniers se trouvent généralement piégés dans des jeux stratégiques ou langagiers qu'ils ont contribué à lancer sans pouvoir les maîtriser du fait, précisément, du débat communautaire.

25. Je reprends ici les termes utilisés par Jean-François Lyotard pour caractériser l'émergence de la postmodernité : " Ainsi la société qui vient relève moins d'une anthropologie newtonienne (comme le structuralisme ou la théorie des systèmes) et davantage d'une pragmatique des particules langagières. Il y a beaucoup de jeux de langage différents, c'est l'hétérogénéité des éléments. Ils ne donnent lieu à institution que par plaques, c'est le déterminisme local ". La condition postmoderne, Les éditions de Minuit, Paris, 1979, p. 8.

26. " Service public, services publics : déclin ou renouveau ", Etudes et documents, Paris, La Documentation française, 1994.

27. " Rapport à Monsieur le Premier Ministre sur le service public ", Paris, La Documentation française, 1996.

28. " Les services d'intérêt général en Europe ", Communication de la Commission, Com (96) 443, 11 septembre 1996. 
29. Il est significatif que la loi de réglementation des télécommunications du 26 juillet 1996 prévoit expressément une " clause de rendez-vous " afin de réviser tous les cinq ans les obligations des opérateurs chargés de mettre en œuvre le service universel du téléphone.

30. C'est ainsi que l'accord social d'EDF-GDF de 1993 fait entrer la notion de contrepartie entre la garantie de l'emploi et " l'acceptation des adaptations nécessaires aux évolutions et changements des métiers ". Sur l'évolution des relations professionnelles au sein des entreprises à statut et les problèmes juridiques que cela soulève, voir Danielle Charles-Le Bihan, " La transformation du jeu social entre les services publics et leurs agents ", Revue française d'administration publique, 80, octobre-décembre 1996, p. 659 et s.

INDEX

Index géographique : Bruxelles, Europe

Mots-clés : construction européenne, politiques publiques, bureaucraties/ théorie des organisations 